\title{
Inhibition of translation in living eukaryotic cells by an RNA G-quadruplex motif
}

\author{
AMIT ARORA, ${ }^{1,2}$ MARIOLA DUTKIEWICZ, ${ }^{1}$ VINOD SCARIA, ${ }^{3}$ MANOJ HARIHARAN, ${ }^{3}$ \\ SOUVIK MAITI, ${ }^{2}$ and JENS KURRECK ${ }^{1,4}$ \\ ${ }^{1}$ Institute for Chemistry and Biochemistry, Free University of Berlin, Berlin, Germany \\ ${ }^{2}$ Proteomics and Structural Biology Unit, Institute of Genomics and Integrative Biology, Delhi, India \\ ${ }^{3}$ GN Ramachandran Knowledge Center for Genome Informatics, Institute of Genomics and Integrative Biology, Delhi, India \\ ${ }^{4}$ Institute of Industrial Genetics, University of Stuttgart, Stuttgart, Germany
}

\begin{abstract}
Guanine-rich sequences can adopt intramolecular four-stranded structures, called G-quadruplexes. These motifs have been intensively investigated on the DNA level, but their overall biological relevance remains elusive. Only recently has research concerning the function of G-quadruplexes in RNAs commenced. Here, we demonstrate for the first time, that an RNA Gquadruplex structure inhibits translation in vivo in eukaryotic cells. We investigated the function of a highly conserved, thermodynamically stable RNA G-quadruplex in the 5'-UTR of the mRNA of the human Zic-1 zinc-finger protein. Using dual luciferase reporter assay, we demonstrate that the Zic-1 RNA G-quadruplex represses protein synthesis inside eukaryotic cells. Quantitative RT-PCR assays confirmed that the reduction of protein synthesis is due to regulation of the translation process and not a consequence of reduced transcription. Western blot analysis revealed that expression of Zic-1 is strongly reduced by a 73 nucleotides-long fragment of the UTR containing the G-quadruplex motif. These structures might add to the more recently discovered elements in untranslated regions of mRNAs that regulate their translation.
\end{abstract}

Keywords: G-quadruplex; RNA structure; Zic-1; translational repression

\section{INTRODUCTION}

Guanine-rich nucleic acids have the potential to form unusual secondary structures comprising of four Hoogsteenpaired coplanar guanines, known as G-quadruplexes (GQ) (Gellert et al. 1962). The best characterized G-rich sequences that are able to fold into quadruplex structures are the capping structures at the ends of eukaryotic chromosomes, the telomeres (Mills et al. 2002; Rhodes et al. 2002; Neidle and Parkinson 2003; Cech 2004). Strong evidence for the existence of G-rich quadruplex structures in vivo was observed by staining of telomeres with quadruplex-specific antibodies (Schaffitzel et al. 2001). Furthermore, selective binding of proteins and small molecules to G-rich mRNAs and telomeric DNA (Darnell et al. 2001;

Reprint requests to: Souvik Maiti, Proteomics and Structural Biology Unit, Institute of Genomics and Integrative Biology, CSIR, Mall Road, Delhi 110 007, India; e-mail: souvik@igib.res.in; fax: +91 011 27667471; or Jens Kurreck, Institute of Industrial Genetics, University of Stuttgart, Allmandring 31, 70569 Stuttgart, Germany; e-mail: jens.kurreck@iig.unistuttgart.de; fax: +4971168566973.

Article published online ahead of print. Article and publication date are at http://www.rnajournal.org/cgi/doi/10.1261/rna.1001708.
Ramos et al. 2003; Granotier et al. 2005; Paeschke et al. 2005) was reported and the formation of DNA GQ structures during transcription was observed (Duquette et al. 2004). Moreover, small molecules that bind to and thereby stabilize DNA GQs have been shown to suppress gene expression of certain proto-oncogenes, hence providing a potential new therapeutic approach (Rangan et al. 2001; Siddiqui-Jain et al. 2002).

Despite the growing body of evidence for the existence of GQs in biological systems (recently reviewed in Patel et al. 2007), their overall functional relevance is still under debate. This is particularly true for the role of DNA GQs, due to the competition between quadruplex formation by one of the two strands and hybridization of the two complementary strands ( $\mathrm{Li}$ et al. 2003; Risitano and Fox 2003; Kumar and Maiti 2005). Unlike DNA, transcribed RNA is exported to the cytoplasm, where it experiences more changes in the concentration of ions and molecular crowding agents than DNA existing solely in the nucleus. This suggests that RNA GQs can act as tuneable devices depending on cellular conditions.

Research in recent years revealed an unexpected relevance of RNA-mediated regulation of protein synthesis at 
the post-transcriptional level, e.g., via the formation of secondary structures like hairpin loops (Kozak 1991), riboswitches (Mandal and Breaker 2004), or small RNAs (Rana 2007). A potential regulative role of four-stranded GQ secondary structures has been suggested for alternative splicing of human pre-mRNA (Gomez et al. 2004; Kostadinov et al. 2006). It has also been shown recently that an RNA G-quadruplex in the $5^{\prime}$-UTR of the NRAS mRNA modulates protein synthesis in an artificial in vitro translation system (Kumari et al. 2007). In another study, a G-quadruplex in the fragile X premutation mRNA was described to influence protein synthesis (Khateb et al. 2007). Quadruplex forming G-rich sequences identified by bioinformatic analysis of UTRs of eukaryotic mRNAs were assembled in the GRS_UTR database (Kikin et al. 2008). Furthermore, Wieland and Hartig (2007) introduced tunable RNA GQ motifs to modulate gene expression in bacteria. Here, we demonstrate for the first time that a GQ motif in the $5^{\prime}$-UTR of an mRNA can inhibit translation in living eukaryotic cells. We investigated the effect of an evolutionarily conserved GQ from the mRNA of the zinc-finger protein of the cerebellum 1 (Zic-1). The motif was found to drastically repress translation without affecting the mRNA level in vivo.

\section{RESULTS AND DISCUSSION}

Bioinformatic analyses have shown that up to 376,000 potential DNA GQs exist in the human genome (Huppert and Balasubramanian 2005; Todd et al. 2005). Since we aimed at investigating a possible regulative role in translation, we were interested in motifs being located in the $5^{\prime}$ UTRs of mRNAs. We employed our previously developed computational search algorithm (Scaria et al. 2006) to identify several stable and highly conserved GQ motifs in the 5'-UTRs of mRNAs. As a first candidate, we chose the mRNA of the zinc-finger protein Zic-1. This protein plays a role in cerebellar development (Aruga et al. 1998) and is also considered to be of pathological relevance, as it was found to be overexpressed in medulloblastoma (26/29 cases), while it was not present in any of the other tumors examined (Yokota et al. 1996; Michiels et al. 1999). The 719-nucleotide-(nt) long Zic-1 5'-UTR contains an RNA GQ motif, which starts 46 nt upstream of the translation start site. This motif is evolutionarily conserved across the human, mouse, chimpanzee, and macaque genes orthologous to $Z i c-1$ (Table 1).

\section{Thermodynamic and structural characterization of Zic-1 RNA quadruplex}

The thermal melting of quadruplex structures can be characterized by an inverse UV transition (Mergny et al. 1998). The UV melting profile of the putative Zic-1 RNA quadruplex sequence in a buffer containing $25 \mathrm{mM} \mathrm{KCl}$ shows hypochromic transition with a characteristic sigmoidal curve (Fig. 1A). Curves for melting and annealing were virtually identical. The $T_{m}$ value was found to be $79^{\circ} \mathrm{C}$. At a higher $\mathrm{KCl}$ concentration $(100 \mathrm{mM})$, the structure could not be unfolded, even at $95^{\circ} \mathrm{C}$, which is indicative of a very stable quadruplex (data not shown). Furthermore, the $T_{m}$ was found to depend on the nature of the monocation. In the presence of $25 \mathrm{mM} \mathrm{NaCl}, T_{m}$ was $15^{\circ} \mathrm{C}$ lower than in the presence of $\mathrm{KCl}$ at the same concentration (data not shown). This finding confirms the well-known fact that GQ motifs are more stable in the presence of potassium ions than in the presence of any other monocation (Rachwal et al. 2007).

Circular dichroism (CD) spectroscopy is a standard technique to analyze structural features of GQ motifs (Kumari et al. 2007; Wieland and Hartig 2007). This method was therefore used to characterize the structural conformation of the putative Zic-1 RNA GQ in terms of parallel or antiparallel topology. As shown in Figure 1B, the Zic-1 GQ motif reveals a positive band at around $263 \mathrm{~nm}$ and a negative band near $240 \mathrm{~nm}$. These features are typical for a parallel-oriented quadruplex structure. RNA quadruplexes generally disfavor the antiparallel fold, since this would require guanosines to adopt in part the syn-conformation, which is highly unfavorable for RNA due to the ribose C3' endo conformation (Tang and Shafer 2006).

In a mutated variant of the Zic-1 GQ RNA various guanosines were replaced by adenosine. To confirm that this RNA does not form a GQ structure, a melting curve

TABLE 1. Conservation of the G-quadruplex forming motif in the $5^{\prime}$-UTR of Zic-1

\begin{tabular}{lrll}
\hline Organism & \multicolumn{1}{c}{ Sequence $^{\text {a }}$} & Accession No. $^{\text {Position }^{\text {b }}}$ \\
\hline Human & GGGUGGGGGGGG_CGGGGGAGGCCGGGG & NM_003412 & -46 \\
Mouse & GGGCGGGUGGGGGGGGCGGGGGAGGCCGGGG & NM_009573.3 & -47 \\
Chimpanzee & GGGUGGGGGGG_CGGGG_AGGCCCGGG & XM_516806.2 & $-172^{\text {c }}$ \\
Macaque & GGGUGGGGGGGG_CGGGGGAGGCCGGGG & XM_001105173.1 \\
Consensus & GGGUGGGGGGG_CGGGG_AGGCC_GGG & & -46 \\
\hline
\end{tabular}

${ }^{a}$ Dashes represent gaps in the alignment. Nucleotides in bold are runs of guanines capable of forming G-quadruplexes.

${ }^{b}$ The position of the first $\mathrm{G}$ of the putative G-quadruplex sequence is given relative to the translation start site.

${ }^{\mathrm{C}}$ The difference in position for Chimpanzee is likely to be due to the incomplete annotation as it is a predicted sequence. 
Zic-1 GQ RNA

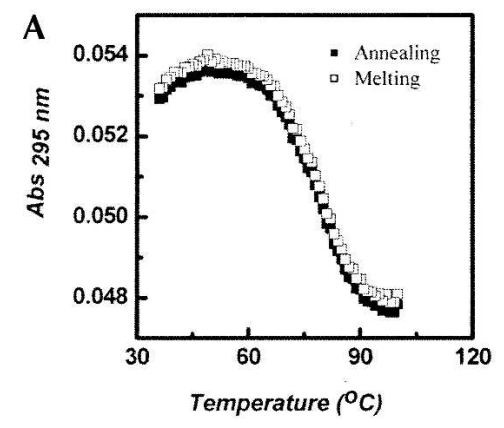

B
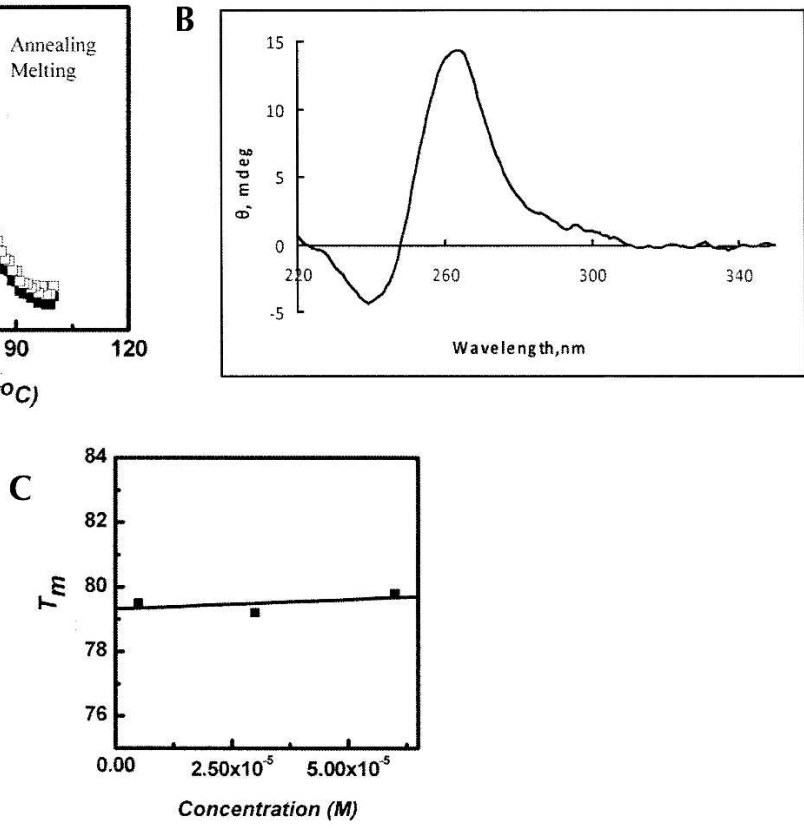

Mutated Zic-1 GQ RNA
D

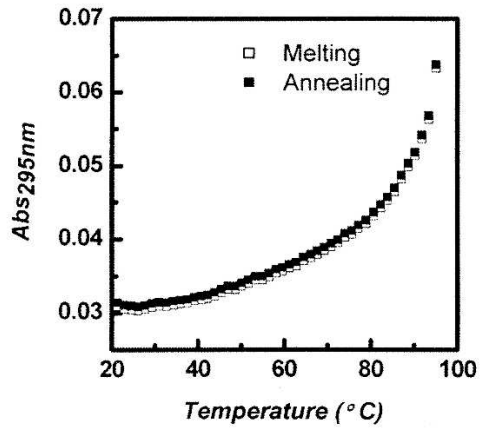

$\mathbf{E}$

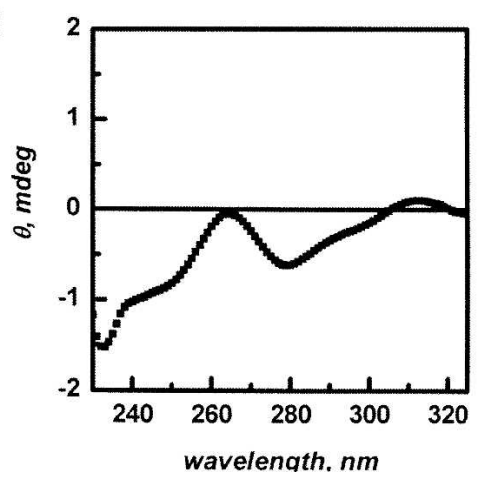

FIGURE 1. Biophysical analysis of the Zic-1 RNA GQ. (A) Melting and annealing curves were recorded at $295 \mathrm{~nm}$. (B) CD spectrum of Zic-1 RNA GQ. (C) Melting temperature $T_{m}$ at different concentrations of the RNA GQ. $(D)$ Melting and annealing curve for the mutated Zic-1 GQ RNA. (E) CD spectrum of the mutated Zic-1 GQ RNA. All experiments were carried out at $25 \mathrm{mM} \mathrm{KCl}$.

(Fig. 1D) at $295 \mathrm{~nm}$ and a CD spectrum (Fig. 1E) were recorded. Neither a distinct hypochromic transition in the melting curve at $295 \mathrm{~nm}$ nor characteristic bands in the CD spectrum were observed, indicating that this sequence is a suitable control that does not form a GQ structure.

To further investigate whether the GQ of the original Zic-1 sequence is formed intermolecularly or intramolecularly, we determined the melting temperature $T_{m}$ at different concentrations of the RNA GQ oligonucleotides (5-60 $\mu \mathrm{M}$ ) in buffer containing $25 \mathrm{mM} \mathrm{KCl}$. As can be seen in Figure $1 \mathrm{C}, T_{m}$ remains virtually unchanged at $79^{\circ} \mathrm{C}$ in the concentration range analyzed. This finding is indicative for the formation of an intramolecular GQ motif. Together, the UV and CD profiles reflect that the G-rich Zic-1 RNA folds into a very stable, parallel intramolecular GQ structure under near physiological $\mathrm{pH}$ and salt conditions.

\section{Inhibition of translation in living human cells}

Our next aim was to investigate the influence of the RNA GQ motif on translation in living eukaryotic cells. Previous studies have shown that GQ motifs in the $5^{\prime}$-UTR of an mRNA can reduce translation in a rabbit reticulocyte lysate in vitro (Kumari et al. 2007). Furthermore, artificial GQ motifs that mask the Shine-Dalgarno sequences were found to suppress gene expression in bacteria (Wieland and Hartig 2007). However, to the best of our knowledge, the question of whether or not RNA GQ structures influence translation in vivo in eukaryotic cells has not yet been addressed.

To clarify this important point, we performed dual luciferase assays in HeLa cells. The psiCHECK-2 vector from Promega allows simultaneous expression of Renilla and firefly luciferase from a single plasmid (Fig. 2A). To evaluate the influence of the Zic-1 RNA GQ on the efficiency of translation, we cloned a 27-base pair-long DNA sequence encoding the GQ motif upstream of the Renilla luciferase. This plasmid was named GQ27 (Fig. 2A). As a control, a mutated version of the GQ was used, in which several guanosines of the original sequence were replaced by adenosines preventing the formation of the GQ structure (Fig. 2A, GQ27m).

The three vector constructs (psiCHECK-2, GQ27, or GQ27m) were transfected into HeLa cells, and $24 \mathrm{~h}$ after transfection, cells were harvested for dual luciferase assays. The ratio of Renilla and firefly luciferase activity in the original psiCHECK-2 control vector was $\sim 30$. Figure $2 \mathrm{~B}$ (black bars) shows the ratios of Renilla and firefly luciferase activity normalized to the value obtained for the unmodified psiCHECK-2 vector. Strikingly, insertion of the Zic-1 RNA GQ27 motif upstream of the Renilla luciferase start codon drastically reduces protein synthesis by $\sim 80 \%$. In contrast, the mutated version GQ27m, which does not form a stable quadruplex structure (Fig. $1 \mathrm{D}, \mathrm{E})$, does not significantly influence translation. The 
A

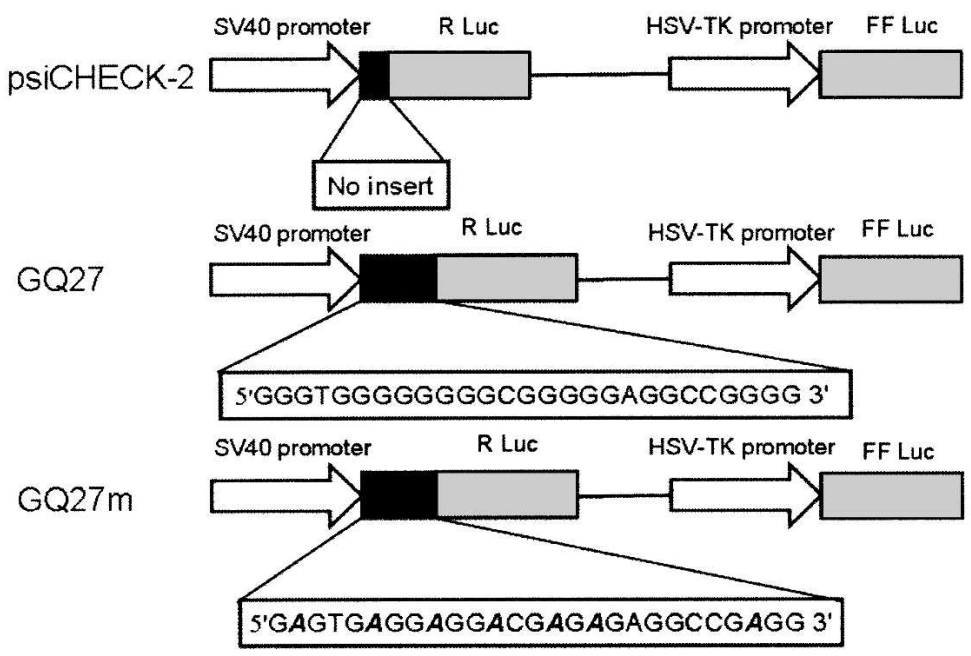

B

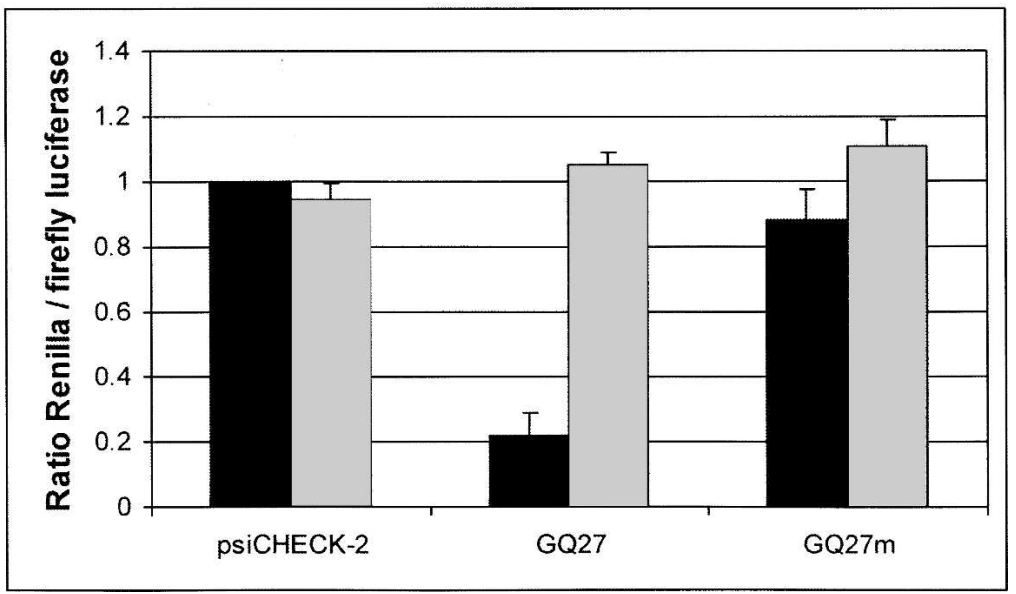

FIGURE 2. (A) Plasmids for the investigation of the influence of the GQ motif on translation. The original psiCHECK-2 vector (Promega) expresses Renilla and firefly luciferase under control of the SV40 and HSV-TK promoter, respectively. The plasmid GQ27 contains the 27-nt-long GQ-motif of the Zic-1 5'-UTR upstream of the start codon. In the control plasmid GQ27m several guanosines were replaced by adenosines (bold and italics). (B) Relative levels of Renilla and firefly luciferase. Protein activity was determined by dual-luciferase reporter assays (black bars). Plasmids were transfected into HeLa cells. Twenty-four hours after transfection cells were harvested and dual-luciferase assays were carried out. The ratios of Renilla and firefly luciferase activity were calculated. The ratio of the control plasmid psiCHECK-2 was set as 1 and the other values were normalized accordingly. Average values and standard deviations obtained from four independent experiments are shown. Relative mRNA levels were determined by real-time PCR (gray bars). Again, HeLa cells were transfected with either of the plasmids and total RNA was isolated after $24 \mathrm{~h} . C_{T}$ values of Renilla and firefly luciferase mRNAs were determined by quantitative RT-PCR. Ratios of Renilla and firefly mRNA levels were calculated as $C_{T}$ (Renilla)/ $C_{T}$ (firefly). Averages and standard deviations of three independent experiments are shown.

extent of translational repression found in living cells is comparable to what was reported for an in vitro translation system before. Balasubramanian and coworkers reported an approximately fourfold decrease in protein synthesis in rabbit reticulocyte lysate caused by a GQ motif in the $5^{\prime}$-UTR of the NRAS mRNA (Kumari et al. 2007).

We next wanted to know whether the GQ motif represses gene expression on the transcriptional or translational level. To this end, we performed realtime PCR assays with lysates from those that were transfected with the empty psiCHECK-2, GQ27 and GQ27m plasmid, respectively. Twenty-four hours after transfection, total RNA was isolated and quantitative RT-PCR assays were performed. As can be seen in Figure 2B (gray bars) almost identical levels were observed for all three constructs, i.e., the GQ motif does not reduce the mRNA level of the Renilla luciferase. We thus conclude that the decrease of protein synthesis is due to repression of translation rather than a consequence of reduced transcription.

\section{Suppresion of Zic-1 protein synthesis in the presence of G-quadruplex motif}

In order to investigate the role of the RNA GQ on the Zic-1 expression, we analyzed synthesis of the Zic-1 protein in the presence or absence of the guanosine-rich sequence upstream of the start codon. Eukaryotic HeLa cells were transfected with a plasmid encoding Zic-1 either in the presence or absence of a 73-nt-long fragment of the natural $5^{\prime}$-UTR containing the GQ-forming sequence. Twenty-four hours after transfection, cells were harvested and lysates for Western blots were prepared. An unspecific band of $\sim 64 \mathrm{kDa}$ is detected in all samples, but no endogenous expression of Zic-1 was observed in untransfected HeLa cells (Fig. 3, lane 1). Similarly, virtually no Zic-1 expression could be detected from plasmids containing the fragment of the 5'-UTR (Fig. 3, lane 2). In sharp contrast, a strong band at the expected size of $\sim 50 \mathrm{kDa}$ was observed in the lysate of cells which were transfected with the plasmid encoding Zic-1 in the absence of the $5^{\prime}$ UTR (Fig. 3, lane 3). These results clearly confirm that the GQ motif strongly represses protein synthesis.

The functional relevance of G-quadruplex structures in living cells is still under investigation. In our present study, 


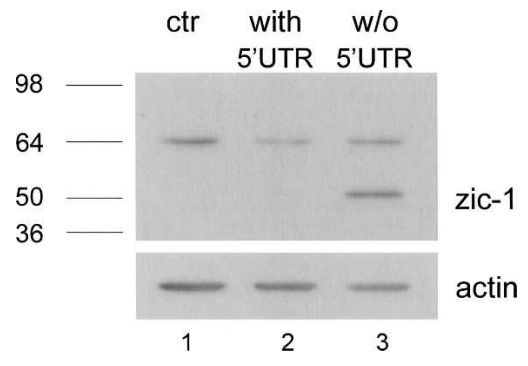

FIGURE 3. Expression of Zic-1 in the presence and absence of the partial 5'-UTR, containing the GQ motif. HeLa cells were transfected with $0.8 \mu \mathrm{g}$ of the pcDNA3.1 plasmid expressing Zic-1 with (lane 2) or without (lane 3) partial 5'-UTR. Cells were lysed $24 \mathrm{~h}$ after transfection. Western blot with Zic-1 specific antibodies was performed as described in the Materials and Methods section. Lane 1 shows the lysate of untransfected cell; an actin blot was performed as a loading control.

we demonstrate that an RNA GQ in the 5'-UTR strongly represses protein synthesis. Since the GQ motif does not influence the mRNA level, we conclude that this effect is due to inhibition of translation. GQ motifs occurring in numerous genes might be a new class of elements in untranslated regions that add to the recently discovered repertoire of regulatory elements in mRNAs like riboswitches and aptazymes. Since a number of low molecular weight substances have been developed that specifically bind to $G$ quadruplexes, these motifs can be considered as an attractive target for small molecular therapeutics.

\section{MATERIALS AND METHODS}

\section{Oligonucleotides}

The following RNA oligonucleotides for spectroscopic studies was purchased from Chemgenes Corp.:

Zic-1 5'-GGGUGGGGGGGGCGGGGGAGGCCGGGG-3'; and Mutated Zic-1 5'-GAGUGAGGAGGACGAGAGAGGCCGAGG-3' .

RNA concentration was determined by UV measurement using an extinction coefficient of $26.63 \times 10^{4} \mathrm{M}^{-1} \mathrm{~cm}^{-1}$ and $28.82 \times 10^{4}$ $\mathrm{M}^{-1} \mathrm{~cm}^{-1}$ for Zic-1 and mutated Zic-1, respectively.

DNA oligonucleotides for PCR and cloning were purchased from TIB MOLBIOL.

\section{Spectroscopy}

For spectroscopic studies, we prepared RNA samples at $5 \mu \mathrm{M}$ concentration in a buffer containing $10 \mathrm{mM}$ sodium cacodylate at

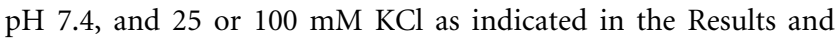
Discussion section. Samples were annealed by heating at $90^{\circ} \mathrm{C}$ for $10 \mathrm{~min}$ and subsequent cooling down to $5^{\circ} \mathrm{C}$. $\mathrm{CD}$ experiments were performed at $20^{\circ} \mathrm{C}$ using a Jasco J-810 spectropolarimeter (Jasco Hachioji, Tokyo, Japan) equipped with a Peltier temperature controller. CD scans were taken in triplicates and their average was calculated. A CD spectrum of the buffer was recorded and subtracted from the spectrum obtained for the RNA-containing solution. Data were zero-corrected at $350 \mathrm{~nm}$.

UV-melting studies were carried out on a Cary 100 UV-visible spectrophotometer (Varian) equipped with a Peltier temperature controller. Samples were heated to $90^{\circ} \mathrm{C}$ and cooled down to $5^{\circ} \mathrm{C}$ at a $0.2^{\circ} \mathrm{C} \min ^{-1}$ temperature gradient, and absorption data recorded at $295 \mathrm{~nm}$ were collected every $0.1 \mathrm{~min}$ on both annealing and melting steps.

\section{Construction of plasmids}

To construct the plasmid GQ27, a synthetic DNA duplex encoding the G-rich sequence of the Zic-1 5'UTR was inserted into the unique NheI restriction site of the psiCHECK-2 plasmid (Promega) upstream of the Renilla luciferase start codon. The following DNA oligonucleotides were used:

\section{GQ27 sense: CTAGCGGGTGGGGGGGGCGGGGGAGGCCGG GGA; and \\ GQ27 antisense: CTAGTCCCCGGCCTCCCCCGCCCCCCCCAC CCG.}

The cloning procedure results in the following mRNA sequence (the GQ motif is underlined; the start codon is given in italics):

\section{5' -AUAGCUAGCGGGUGGGGGGGGCGGGGGAGGCCGGGGA GCUAGCCACCAUG-3'.}

The control vector GQ27m containing the GQ motif with several $\mathrm{G}$ to A substitutions was generated with the following oligonucleotides:

GQ27m sense: CTAGCGAGTGAGGAGGACGAGAGAGGCCGA GGA; and

GQ27m antisense: CTAGTCCTCGGCCTCTCTCGTCCTCCTCA CTCG.

A Zic-1 cDNA clone with a partial 5'-UTR containing the GQ forming region was obtained from Bio Cat as the pCR4-TOPO vector. The Zic-1 cDNA with or without the partial 5'UTR was subcloned into the eukaryotic expression vector pcDNA3.1.

\section{Cell culture}

$\mathrm{HeLa}$ cells were grown at $37^{\circ} \mathrm{C}$ in a humidified atmosphere containing $5 \% \mathrm{CO}_{2}$ in Dulbecco's modified Eagles medium (DMEM) supplemented with $10 \%$ heat-inactivated fetal calf serum (FCS), $2 \mathrm{mM}$ glutamine, nonessential amino acids, and the antibiotics penicillin and streptomycin.

\section{Transient transfection and dual luciferase reporter assay}

Transfection was carried out in 24-well plates using LipofectAMINE 2000 (Invitrogen) according to the manufacturer's instructions. Twenty-four hours after transfection, cells were resuspended in passive lysis buffer (Promega). The firefly and Renilla luciferase activities are measured using the Dual-luciferase Reporter Assay kit (Promega) according to the manufacturer's instructions with a luminometer (TD 20/20 Luminometer, Turner BioSystems). 


\section{Quantitative real-time PCR assay}

For quantitative analysis of the mRNA levels, total cellular RNA was isolated from transfected cells using the RNeasy mini kit along with RNase-free DNase to remove any traces of DNA contamination (QIAGEN). The first-strand cDNA was synthesized with M-MLV reverse transcriptase (Promega). cDNA was then subjected to quantitative real-time PCR, using a SYBR-Green PCR Master Mix (Applied Biosystems) and the ABI Prism 7300 Detection system (Applied Biosystems).

\section{Western blot}

Zic-1 protein was immunoblotted with an anti-Zic-1 primary antibody (ab24204, Abcam). Actin was subsequently probed as an internal loading control (MAB1501R, Chemicon). Goat antirabbit and goat antimouse secondary antibodies conjugated with horseradish peroxidase (HRP) were used for Zic-1 and actin detection, respectively. Antigen-antibody complexes were visualized by the ECL system (Pierce).

\section{ACKNOWLEDGMENTS}

The authors thank Diana Rothe and Denise Werk for their help, and Volker A. Erdmann for fruitful discussions. A.A. is a fellow of the German Academic Exchange Service (DAAD) and a Senior Research Fellow (SRF) of the University Grants Commission (UGC), India. M.D. is a fellow of the German Academic Exchange Service (DAAD). V.S. and M.H. thank the Council for Scientific and Industrial Research (CSIR), India, for a Senior Research Fellowship. Financial support from the Free University Berlin, the Fonds der Chemischen Industrie, and Council for Scientific and Industrial Research (CSIR), India, is gratefully acknowledged.

Received January 11, 2008; accepted April 5, 2008.

\section{REFERENCES}

Aruga, J., Minowa, O., Yaginuma, H., Kuno, J., Nagai, T., Noda, T., and Mikoshiba, K. 1998. Mouse Zicl is involved in cerebellar development. J. Neurosci. 18: 284-293.

Cech, T.R. 2004. Beginning to understand the end of the chromosome. Cell 116: 273-279.

Darnell, J.C., Jensen, K.B., Jin, P., Brown, V., Warren, S.T., and Darnell, R.B. 2001. Fragile X mental retardation protein targets G quartet mRNAs important for neuronal function. Cell 107: 489499.

Duquette, M.L., Handa, P., Vincent, J.A., Taylor, A.F., and Maizels, N. 2004. Intracellular transcription of G-rich DNAs induces formation of G-loops, novel structures containing G4 DNA. Genes \& Dev. 18: 1618-1629.

Gellert, M., Lipsett, M.N., and Davies, D.R. 1962. Helix formation by guanylic acid. Proc. Natl. Acad. Sci. 48: 2013-2018.

Gomez, D., Lemarteleur, T., Lacroix, L., Mailliet, P., Mergny, J.L., and Riou, J.F. 2004. Telomerase down-regulation induced by the Gquadruplex ligand 12459 in A549 cells is mediated by hTERT RNA alternative splicing. Nucleic Acids Res. 32: 371-379.

Granotier, C., Pennarun, G., Riou, L., Hoffschir, F., Gauthier, L.R., De Cian, A., Gomez, D., Mandine, E., Riou, J.F., Mergny, J.L., et al. 2005. Preferential binding of a G-quadruplex ligand to human chromosome ends. Nucleic Acids Res. 33: 4182-4190.
Huppert, J.L. and Balasubramanian, S. 2005. Prevalence of quadruplexes in the human genome. Nucleic Acids Res. 33: 29082916.

Khateb, S., Weisman-Shomer, P., Hershco-Shani, I., Ludwig, A.L., and Fry, M. 2007. The tetraplex (CGG) ${ }_{n}$ destabilizing proteins hnRNP A2 and CBF-A enhance the in vivo translation of fragile X premutation mRNA. Nucleic Acids Res. 35: 57755788.

Kikin, O., Zappala, Z., D’Antonio, L., and Bagga, P.S. 2008. GRSDB2 and GRS_UTRdb: Databases of quadruplex forming G-rich sequences in pre-mRNAs and mRNAs. Nucleic Acids Res. 36: D141-D148.

Kostadinov, R., Malhotra, N., Viotti, M., Shine, R., D’Antonio, L., and Bagga, P. 2006. GRSDB: A database of quadruplex forming G-rich sequences in alternatively processed mammalian pre-mRNA sequences. Nucleic Acids Res. 34: D119-D124.

Kozak, M. 1991. Structural features in eukaryotic mRNAs that modulate the initiation of translation. J. Biol. Chem. 266: 1986719870.

Kumar, N. and Maiti, S. 2005. The effect of osmolytes and small molecule on Quadruplex-WC duplex equilibrium: A fluorescence resonance energy transfer study. Nucleic Acids Res. 33: 67236732.

Kumari, S., Bugaut, A., Huppert, J.L., and Balasubramanian, S. 2007. An RNA G-quadruplex in the $5^{\prime}$-UTR of the NRAS protooncogene modulates translation. Nat. Chem. Biol. 3: 218-221.

Li, W., Miyoshi, D., Nakano, S., and Sugimoto, N. 2003. Structural competition involving G-quadruplex DNA and its complement. Biochemistry 42: 11736-11744.

Mandal, M. and Breaker, R.R. 2004. Gene regulation by riboswitches. Nat. Rev. Mol. Cell Biol. 5: 451-463.

Mergny, J.L., Phan, A.T., and Lacroix, L. 1998. Following G-quartet formation by UV-spectroscopy. FEBS Lett. 435: 74-78.

Michiels, E.M., Oussoren, E., Van Groenigen, M., Pauws, E., Bossuyt, P.M., Voute, P.A., and Baas, F. 1999. Genes differentially expressed in medulloblastoma and fetal brain. Physiol. Genomics 1: 83-91.

Mills, M., Lacroix, L., Arimondo, P.B., Leroy, J.L., Francois, J.C., Klump, H., and Mergny, J.L. 2002. Unusual DNA conformations: Implications for telomeres. Curr. Med. Chem. Anticancer Agents 2: 627-644.

Neidle, S. and Parkinson, G.N. 2003. The structure of telomeric DNA. Curr. Opin. Struct. Biol. 13: 275-283.

Paeschke, K., Simonsson, T., Postberg, J., Rhodes, D., and Lipps, H.J. 2005. Telomere end-binding proteins control the formation of G-quadruplex DNA structures in vivo. Nat. Struct. Mol. Biol. 12: 847-854.

Patel, D.J., Phan, A.T., and Kuryavyi, V. 2007. Human telomere, oncogenic promoter and 5'-UTR G-quadruplexes: Diverse higher order DNA and RNA targets for cancer therapeutics. Nucleic Acids Res. 35: 7429-7455.

Rachwal, P.A., Findlow, I.S., Werner, J.M., Brown, T., and Fox, K.R. 2007. Intramolecular DNA quadruplexes with different arrangements of short and long loops. Nucleic Acids Res. 35: 42144222.

Ramos, A., Hollingworth, D., and Pastore, A. 2003. G-quartetdependent recognition between the FMRP RGG box and RNA. RNA 9: 1198-1207.

Rana, T.M. 2007. Illuminating the silence: Understanding the structure and function of small RNAs. Nat. Rev. Mol. Cell Biol. 8: 2336.

Rangan, A., Fedoroff, O.Y., and Hurley, L.H. 2001. Induction of duplex to G-quadruplex transition in the c-myc promoter region by a small molecule. J. Biol. Chem. 276: 4640-4646.

Rhodes, D., Fairall, L., Simonsson, T., Court, R., and Chapman, L. 2002. Telomere architecture. EMBO Rep. 3: 1139-1145.

Risitano, A. and Fox, K.R. 2003. Stability of intramolecular DNA quadruplexes: Comparison with DNA duplexes. Biochemistry 42: 6507-6513. 
Scaria, V., Hariharan, M., Arora, A., and Maiti, S. 2006. Quadfinder: Server for identification and analysis of quadruplex-forming motifs in nucleotide sequences. Nucleic Acids Res. 34: W683W685.

Schaffitzel, C., Berger, I., Postberg, J., Hanes, J., Lipps, H.J., and Pluckthun, A. 2001. In vitro generated antibodies specific for telomeric guanine-quadruplex DNA react with Stylonychia lemnae macronuclei. Proc. Natl. Acad. Sci. 98: 85728577.

Siddiqui-Jain, A., Grand, C.L., Bearss, D.J., and Hurley, L.H. 2002. Direct evidence for a G-quadruplex in a promoter region and its targeting with a small molecule to repress c-MYC transcription. Proc. Natl. Acad. Sci. 99: 11593-11598.
Tang, C.F. and Shafer, R.H. 2006. Engineering the quadruplex fold: Nucleoside conformation determines both folding topology and molecularity in guanine quadruplexes. J. Am. Chem. Soc. 128: 5966-5973.

Todd, A.K., Johnston, M., and Neidle, S. 2005. Highly prevalent putative quadruplex sequence motifs in human DNA. Nucleic Acids Res. 33: 2901-2907.

Wieland, M. and Hartig, J.S. 2007. RNA quadruplex-based modulation of gene expression. Chem. Biol. 14: 757-763.

Yokota, N., Aruga, J., Takai, S., Yamada, K., Hamazaki, M., Iwase, T., Sugimura, H., and Mikoshiba, K. 1996. Predominant expression of human zic in cerebellar granule cell lineage and medulloblastoma. Cancer Res. 56: 377-383. 

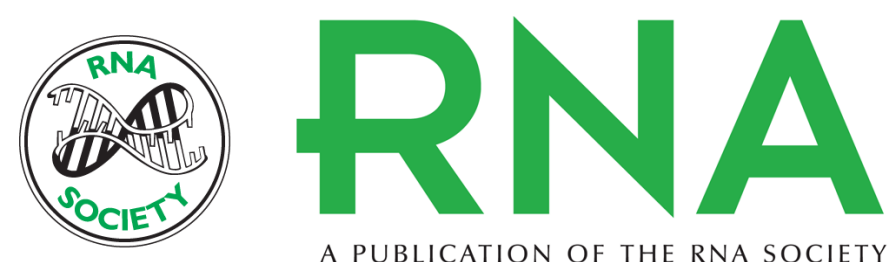

A PUBLICATION OF THE RNA SOCIETY

\section{Inhibition of translation in living eukaryotic cells by an RNA G-quadruplex motif}

Amit Arora, Mariola Dutkiewicz, Vinod Scaria, et al.

RNA 2008 14: 1290-1296

References This article cites 35 articles, 10 of which can be accessed free at: http://rnajournal.cshlp.org/content/14/7/1290.full.html\#ref-list-1

\section{License}

Email Alerting Receive free email alerts when new articles cite this article - sign up in the box at the Service top right corner of the article or click here. 